and infectious disease excesses noted in the technicians was similar to the findings of a large scale survey of medical laboratory workers. $^{3}$

The distinction between hazard and risk from occupational ill health is now the basis of the recently introduced Control of Substances Hazardous to Health Regulations $(1988),{ }^{8}$ which includes a requirement to assess the risks associated with exposure to microbiological as well as chemical agents in the workplace. Epidemiological investigations based on occupational sickness absence records such as this may assist occupational health staff in making such assessments.
This study was carried out with funding from the Department of Health. We are grateful to all post mortem staff and coroners' officers who completed health questionnaires.

\section{Morris SN. Tuberculosis as an occupational hazard during medical training. Am Rev Tuberculosis 1946;54:140-58. \\ 2 Reid DD. Incidence of tuberculosis among workers in medical laboratories. $\mathrm{Br}$ Med J 1957;ii:1-14. \\ 3 Harrington JM, Shannon HS. Incidence of tuberculosis, hepatitis, brucellosis and shigellosis in British medical laboratory workers. $\mathrm{Br} \mathrm{Med} J$ 1976;1:759-62. \\ 4 Grist NR, Emslie JAN. Infections in British clinical laboratories, 1984-85. J Clin Pathol 1987;40:826-9. \\ 5 Grist NR, Emslie JAN. Infections in British clinical laboratories, 1986-87. J Clin Pathol 1989;42:677-81. \\ 6 Babb J, Hall AJ, Marlin R, Ayliffe GAJ. Bacteriological sampling of post mortem rooms. J Clin Pathol 1989;42: 682-8. \\ 7 Office of Population Censuses and Surveys. General house- hold survey. London: HMSO, 1985. \\ 8 Department of Health. Control of Substances Hazardous to} Health Regulations. London: HMSO, 1988.

\title{
Multipoint microbiological assay for detecting $\beta$-lactamase
}

\author{
K J Thickett, T G Winstanley
}

\begin{abstract}
A multipoint microbiological assay for determining $\beta$-lactamase production by clinical isolates of bacteria was evaluated. With strains of Haemophilus influenzae, Neisseria gonorrhoeae, and Branhamella catarrhalis there was excellent correlation between results obtained using this method and those obtained using the chromogenic cephalosporin reference method. The multipoint method is an inexpensive yet reliable adjunct to conventional susceptibility testing methods.
\end{abstract}

The susceptibility testing of micro-organisms such as Branhamella catarrhalis, Haemophilus influenzae, and Neisseria gonorrhoeae using the agar-dilution method has been described. ${ }^{1}$ One drawback of this method is that traditional indicators of $\beta$-lactamase activityreduced zone size and increased colony size at zone edges around antibiotic discs-are absent. We believe $B$ catarrhalis poses a particular problem (unpublished observations). Although around $50 \%$ of strains are resistant to ampicillin as indicated by $\beta$-lactamase production, ${ }^{2}$ many appear susceptible using the accepted ampicillin "break-point" of $1 \mathrm{mg} / 1 .^{3}$ With $B$ catarrhalis, therefore, it is for $\beta$-lactamase detection. Hydrolysis of the $\beta$ lactam bond yields a dibasic acid which can be detected by a change in $\mathrm{pH}$-the acidometric method $^{4}$ or by the ability to reduce iodinethe iodometric method. ${ }^{5}$ With the chromogenic cephalosporin, ${ }^{6}$ an electron shift in the cephalosporin molecule yields a coloured product. Loss of antibacterial activity may also be detected by microbiological assays which use susceptible bacteria to detect breakdown of $\beta$-lactam antibiotics by organisms which are applied by streaking, or on membranes or filter paper discs. $^{7}$ The origins of such assays lie in clinical observations of "indirect pathogenicity" 8 Of these methods, the microbiological assay has proved the easiest to adapt to multipoint methodology.

\section{Methods}

Diagnostic Sensitivity Test agar (DST:Oxoid) was supplemented with lysed horse blood $(7 \%)$ and nicotinamide adenine dinucleotide (Boehringer Mannheim; $20 \mathrm{mg} / \mathrm{l}$ ) to facilitate growth of fastidious organisms. An antiswarming agent (1-4-Nitrophenl-glycerol: $55 \mathrm{mg} / \mathrm{l})$ and penicillin $(0.02 \mathrm{mg} / \mathrm{l})$ were added and the plate was surface seeded with Staphylococcus aureus (NCTC 6571: $10^{5} \mathrm{cfu} / \mathrm{ml}$ ). This plate was inoculated with the bacteria under test after the conventional antibiotic "break-point" set had been inoculated. Overnight incubation in $7 \%$ carbon dioxide showed a "satellite" of growth around organisms producing $\beta$-lactamase (figure).

To assess the reliability of the multipoint method we compared it with the chromogenic imperative to determine whether $\beta$-lactamase is produced if an agar dilution susceptibility testing method is to be used. Detection of $\beta$-lactamase production in $H$ influenzae and $N$ gonorrhoeae also has important clinical implications.

Diagnostic laboratories use four methods 
Control plate (left) and $\beta$-lactamase detection plate (right) showing "satellite" effect around $\beta$-lactamase producing colonies.

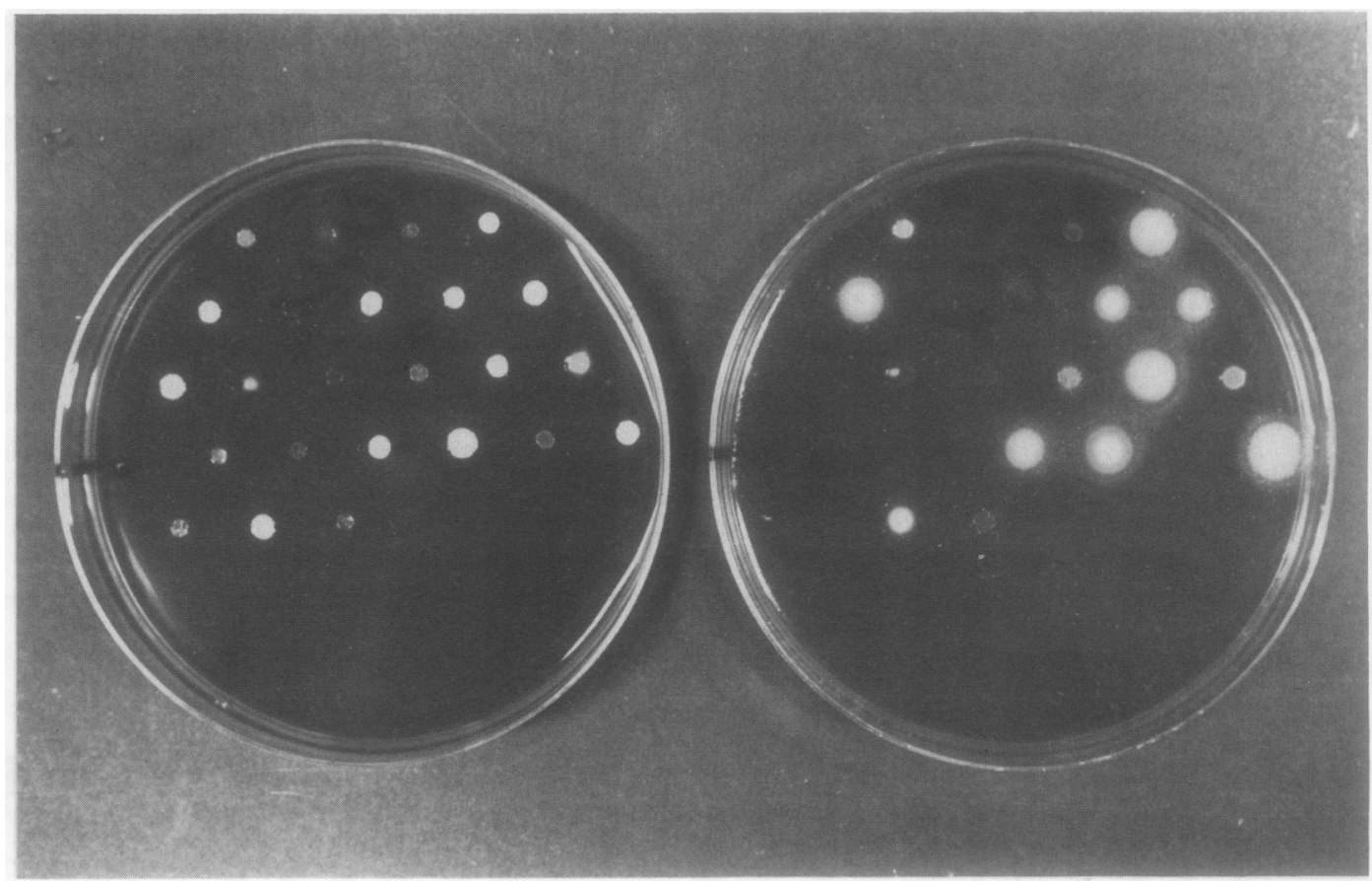

cephalosporin method for detection of $\beta$ lactamase.

Two drops of chromogenic cephalosporin solution ( $500 \mathrm{mg} / \mathrm{l}$; Oxoid) were placed in each well of a 96 well mirotitration tray. Three or four colonies of an overnight culture of the organism to be tested were suspended in $1 \mathrm{ml}$ saline, and one drop of this was added to one well of the microtitration tray. The tray was incubated at room temperature and observed after one hour for positive results. The multipoint method was performed as described above using the same suspension. Positive and negative controls were used for both methods.

\section{Results}

We tested $B$ catarrhalis (60 strains), $H$ influen$z a e$ (60 strains), and $N$ gonorrhoeae (30 strains) and found complete agreement between methods.

\section{Discussion}

The multipoint microbiological assay for detection of $\beta$-lactamase offers an inexpensive, easily controlled, yet reliable method for the detection of $\beta$-lactamase production, which can be added to present working protocols with the minimum of disruption. Plates can be stored at $+4{ }^{\circ} \mathrm{C}$ for two weeks without deterioration in the quality of the reaction.

We are currently undertaking further work to investigate if it is possible to obtain a substrate profile of the $\beta$-lactamase detected by incorporating different $\beta$-lactam antibiotics into the plates. We are also investigating the possibility of modifying the technique to detect other antibiotic inactivating enzymes.

1 National Committee for Clinical Laboratory Standards. Methods for dilution antimicrobial susceptibility tests for bacteria that grow aerobically. NCCLS Document M7-T2, Vol 8, No 8. Villanova, PA: NCCLS, 1989.

2 Winstanley TG, Spencer RC. Moraxella catarrhalis: antibiotic susceptibility with special reference to trimethoprim. J Antimicrob Chemother 1986;18:425-31.

3 Report by a working party of the British Society for Antimicrobial Chemotherapy. Breakpoint in in-vitro antibiotic sensitivity testing. J Antimicrob Chemother 1988;21:701-10.

4 Turnsberry C, Kirven LA. Ampicillin resistance in Haemophilus influenzae as determined by a rapid test for Betalactamase. Antimicrob Agents Chemother 1974;6:653-4.

5 Foley JM, Perret CJ. Screening bacterial colonies for penicillinase production. Nature 1962;195:287-8. method for detection of beta-lactamase by using a chromogenic cephalosporin substrate. Antimicrob Agents Chemother 1972;1:283-8.

7 Lee WS, Komary L. New method for detecting in-vitro inactivation of penicillins by Haemophilus influenzae and Staphylococcus aureus. Antimicrob Agents Chemother 1976;10:564-6.

8 Maddocks JL, May JR. "Indirect pathogenicity" of penicillinase-producing enterobacteria in chronic bronchial infections. Lancet 1969;i:793.
6 O'Callaghan CH, Morris A, Kirby SM, Shingler AH. Novel 